\title{
Nonpustular annular psoriasis: a variant of annular pustular psoriasis but without clinical or histopathological evidence of pustule formation
}

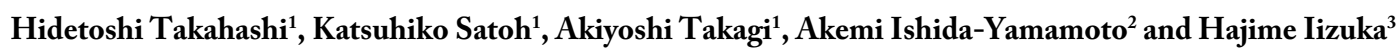 \\ ${ }^{1}$ Takagi Dermatological Clinic, Japan \\ ${ }^{2}$ Department of Dermatology, Asahikawa Medical University, Japan \\ ${ }^{3}$ Department of Dermatology, Housui Medical Center, Japan
}

\section{Case report}

Nonpustular annular psoriasis is a rare form of psoriasis with clinically annular ring-shaped configuration [1]. In contrast to recurrent circinate erythematous psoriasis (Psoriasis a type d'erytheme circine recidivant de Bloch) [2], which is the mildest form of pustular psoriasis, no evidence of histopathological Kogoi's spongiform pustule formation is detected.

A 57-year-old Japanese woman had a 4-year history of annular erythematous plaques on her four extremities (Figure 1A). She denied either pustule or vesicle formation during the course. Physical examination revealed slightly elevated $5-10 \mathrm{~cm}$-sized annular erythematous plaques accompanied with small lamellar scales without pustules. There were no typical non-annular plaque lesions of psoriasis. The annular lesions had been improved by corticosteroid and active vitamin D3 ointments. She had no family history of psoriasis or specific drug intake. Skin biopsies were taken from the infiltrative edge of annular lesions on her extremities. Histopathology showed typical features of psoriasis (Figure 1B), but no evidence of Kogoj's spongiform pustule was detected. Furthermore, the histopathological examination of sections from 3 skin biopsies did not show any infiltration of neutrophils. There was no coat-sleeve-like perivascular cuffing of mononuclear cell infiltration. Laboratory study including full blood cell count and biochemical investigation were normal except for moderate increase in cholesterol and triglyceride level. Serum tests for syphilis, rheumatoid factor, anti-nuclear antibody, SS-A, SS-B, antibodies, HBs antigen and HCV were all negative or within normal

A

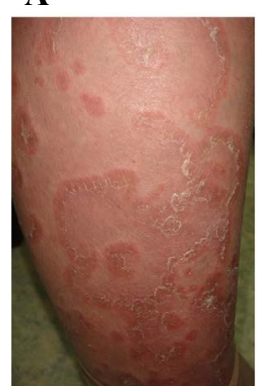

B

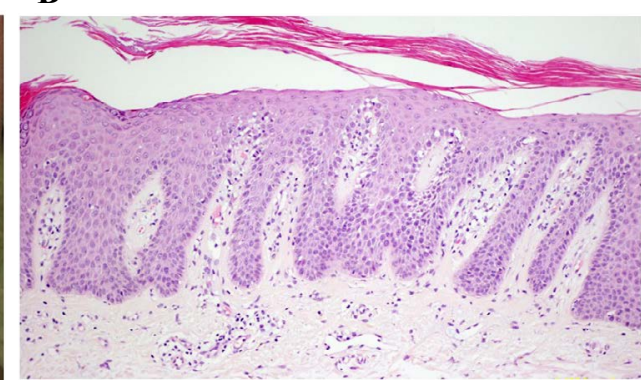

Figure 1. (a) Numerous well-demarcated, annular erythematous plaques on the right lower extremity. Clinically nopustule formation is detected. (b) Histopathology of the scaly edge of the lesion on the right forearm showing hyperplasia and elongated dermal papillae with mononuclear cell infiltration.No Kogoj's spongiform pustule is detected. Magnification is $200 X$. limits. $\mathrm{KOH}$ preparation was negative for fungal infection. The patient was treated with daily application of triamcinolone acetomide $0.1 \%$ and maxacalcitol $(25 \mu \mathrm{g} / \mathrm{g})$ ointments. Her skin lesions gradually improved and no new lesions were observed during the last 6 months. The patient was diagnosed as nonpustular annular psoriasis.

Differential diagnosis of the case includes recurrent circinate erythematous psoriasis (Bloch), circinate annular pustular psoriasis, pityriasis rosea, Sjogren's syndrome, subacute cutaneous lupus erythematosus, tinea corporis, erythema gyratum repens (EGR) and erythema annulare centrifigum. These can be differentiated by laboratory investigation and histopathological analysis. Specifically recurrent circinate erythematous psoriasis (Bloch), though clinically similar, is characterized by the presence of histopathological Kogoj's spongiform pustule formation. Clinical feature of EGR is characteristic and shows rapidly migrating erythema composed of concentric rings forming a wood-grain pattern. It is very difficult to rule out erythema gyratum repens, as it occasionally is not due to a malignancy or the malignancy may be found much later. Thus our case should be under close follow up.

Furthermore, the patient had no evidence of internal malignancy. Thus we excluded the diagnosis of EGR. Erythema annulare centrifugum was excluded by histological findings showing no coatsleeve-like mononuclear cell infiltration.

Previous report indicates that 5 out of 15 annular pustular psoriasis cases, which is recurrent circinate erythematous psoriasis (Bloch), were not accompanied by clinical pustule formation [3]. However, histopathological studies consistently showed spongiform pustules in all these cases [3]. Terunuma et al. [4] and Guill et al. [1] reported three cases of nonpustular annular psoriasis, and suggested that annular psoriasis has features of both typical plaque-type psoriasis and pustular psoriasis, and regarded as an intermediate disease entity of the two. Thus careful follow up of this case should be performed for the possible

Correspondence to: Hidetoshi Takahashi, Takagi Dermatological Clinic, Nishi 3 minami 4-16, Obihiro, Hokkaido 0800013, Japan, Tel: +81-155-25-6733; Fax: +81-155-25-0308; E-mail: htakahashi@cpost.plala.or.jp

Key words: annular psoriasis, Japanese, nonpustular, pustular psoriasis, treatment

Received: August 21, 2016; Accepted: September 09, 2016; Published: September 12, 2016 
Takahashi H (2016) Nonpustular annular psoriasis: a variant of annular pustular psoriasis but without clinical or histopathological evidence of pustule formation

evolution of pustular psoriasis in the future.

\section{References}

1. Guill CL, Hoang MP, Carder KR (2005) Primary annular plaque-type psoriasis. Pediatric Dermatology 22: 15-18. [Crossref]

2. Bazex A, Dupie A, Christol B et al. (1967) Psoriasis a type d'erytheme circine recidivant de Bloch. Bull Soc Fr Dermatol Syphiligr 74: 689-695

3. Rajka G, Thune PO (1979) On erythema annulare centrifugume-type psoriasis. Acta Derm Venereol (Stockh) 59(suppl 88): 143-145. [Crossref]

4. Terunuma A, Takahashi K, Sakakibara A, Aiba S, Tagami H (1992) Annular psoriasiform eruption with lymphocytic infiltration of the epidermis: a variant of acute psoriasis. Dermatology 185: 156-159. [Crossref]

Copyright: (O2016 Takahashi H. This is an open-access article distributed under the terms of the Creative Commons Attribution License, which permits unrestricted use, distribution, and reproduction in any medium, provided the original author and source are credited. 